\title{
Avaliação do estado nutricional e correlação com complicações cirúrgicas em pacientes idosos submetidos a tratamento cirúrgico de fratura do fêmur proximal $^{*}$
}

\section{Evaluation of Nutritional Status and Correlation with Postoperative Complications in Elderly Patients Submitted to Surgical Treatment of Proximal Femoral Fractures}

\author{
Tiane Raquel da Silva Dias ${ }^{1}$ Bruno Bellaguarda Batista ${ }^{1}$ Rafael Wei Min Leal Chang ${ }^{10}$ \\ Jorge Enrique Acosta Noriega ${ }^{1}$ Giuseppe Lemos Pertoti de Figueiredo ${ }^{1}$ \\ 1 Serviço de Ortopedia e Traumatologia, Hospital Universitário \\ Getúlio Vargas (HUGV), Universidade Federal do Amazonas (UFAM), \\ Manaus, AM, Brasil \\ Rev Bras Ortop 2021;56(1):104-108.

\begin{abstract}
Endereço para correspondência Rafael Wei Min Leal Chang, Serviço de Ortopedia e Traumatologia, Hospital Universitário Getúlio Vargas (HUGV), Universidade Federal do Amazonas (UFAM), Rua Tomas de Vila Nova, 4, Nossa Sra. das Gracas, Manaus, AM, 69020-170, Brasil (e-mail: rafchang@gmail.com).
\end{abstract}

\section{Resumo \\ Palavras-chave \\ - complicações pós- operatórias \\ - desnutrição \\ - fraturas do fêmur}

Objetivo Avaliar e correlacionar o estado nutricional com possíveis complicações no pós-operatório imediato de pacientes submetidos a tratamento cirúrgico de fraturas de fêmur proximal.

Métodos Estudo transversal prospectivo analítico e de abordagem quantitativa, tendo como população-alvo pacientes com idade igual ou superior a 60 anos, internados em uma instituição hospitalar no Amazonas, submetidos a tratamento cirúrgico de fraturas de fêmur proximal. Foram avaliadas as complicações cirúrgicas no pós-operatório imediato e sua relação com o estado nutricional através da mini avaliação nutricional (MAN), assim como a mensuração da contagem de linfócitos e albumina e a sua correlação com o tempo de internação.

Resultados A amostra foi composta por 19 pacientes idosos, de ambos os sexos, com média de idade de 70,8 anos. A maioria dos pacientes $(68,4 \%)$ eram do sexo feminino. Por meio da variável antropométrica índice de massa corporal (IMC), identificou-se a presença de desnutrição em 15,8\% dos pacientes e, por meio da MAN, de 31,6\%. Na avaliação do cálculo da contagem total de linfócitos, $100 \%$ da amostra apresentou associação positiva com desnutrição em graus variáveis e, fazendo-se uso da albumina

\footnotetext{
Trabalho desenvolvido no Serviço de Ortopedia e Traumatologia, Hospital Universitário Getúlio Vargas (HUGV, Universidade Federal do Amazonas (UFAM), Manaus, AM, Brasil.
}

recebido

28 de Novembro de 2019

aceito

16 de Setembro de 2020
DOI https://doi.org/ $10.1055 / \mathrm{s}-0040-1721365$ ISSN 0102-3616.
(C) 2021. Sociedade Brasileira de Ortopedia e Traumatologia. All rights reserved.

This is an open access article published by Thieme under the terms of the Creative Commons Attribution-NonDerivative-NonCommercial-License, permitting copying and reproduction so long as the original work is given appropriate credit. Contents may not be used for commercial purposes, or adapted, remixed, transformed or built upon. (https://creativecommons.org/ licenses/by-nc-nd/4.0/)

Thieme Revinter Publicações Ltda., Rua do Matoso 170, Rio de Janeiro, RJ, CEP 20270-135, Brazil 


\begin{abstract}
Keywords

- postoperative complications

- malnutrition

- femoral fractures

Objective This study aims to evaluate and correlate the nutritional status with potential complications during the immediate postoperative period of elderly patients surgically treated for proximal femoral fractures.

Methods A prospective, cross-sectional analytical study with a quantitative approach, targeting patients aged 60 years old or more who were admitted to a hospital in Amazonas, Brazil, for surgical treatment of proximal femoral fractures. Surgical complications during the immediate postoperative period and their relationship with the nutritional status of the patient were determined using the mini nutritional assessment (MNA); in addition, the lymphocyte numbers and serum albumin levels were determined and correlated with the length of hospital stay.

Results The sample consisted of 19 elderly patients, of both genders, with a mean age of 70.8 years. Most of the subjects (68.4\%) were female. Malnutrition was diagnosed in $15.8 \%$ of the subjects using the body mass index (BMI) as, an anthropometric variable, and the MNA identified $31.6 \%$ of the subjects with malnutrition. Regarding total lymphocyte counts, $100 \%$ of the sample showed a positive association with malnutrition in varying degrees; using serum albumin level as a parameter, malnutrition was identified in $89.4 \%$ of the subjects. Malnourished patients had the highest average length of stay. Surgical complications as surgical site infections occurred in $10.5 \%$ of the patients at nutritional risk.

Conclusion This study revealed a higher rate of postoperative complications in elderly patients diagnosed with malnutrition.
\end{abstract}

sérica como parâmetro, a desnutrição foi identificada em $89,4 \%$. Os pacientes desnutridos apresentaram a maior média de tempo de internação. As complicações cirúrgicas, por infecções do sítio cirúrgico, ocorreram em 10,5\% nos pacientes em risco nutricional.

Conclusão Neste estudo, observou-se maior índice de complicações no pós-operatório em idosos diagnosticados com desnutrição.

\section{Introdução}

O estado nutricional está entre as principais preocupações na saúde dos idosos. Com o envelhecimento, mudanças fisiológicas, metabólicas e na capacidade funcional resultam na alteração das necessidades calóricas. O mais importante destes distúrbios observado nos idosos é a desnutrição, que está associada ao aumento da mortalidade e da susceptibilidade às infecções e à redução da qualidade de vida. Esta, entretanto, frequentemente se insere no contexto de outras modificações orgânicas verificadas ao longo do processo de envelhecimento, deixando de ser diagnosticada. ${ }^{1}$

A desnutrição aumenta o risco de desenvolvimento de uma variedade de condições, entre elas: anemia, úlceras de pressão, fraturas ósseas, fragilidade, déficit cognitivo, desidratação, hipotensão ortostática e disfunção imune. ${ }^{2}$ Podese considerar como os principais indicadores de desnutrição nos idosos: a perda de peso involuntária de 5\% em 1 mês, 7,5\% em 3 meses e/ou 10\% em 6 meses; o baixo peso para a altura, inferior a $20 \%$ do peso corporal ideal; o índice de massa corporal (IMC) menor que $22 \mathrm{~kg} / \mathrm{m}^{2}$; a albumina sérica inferior a $3,5 \mathrm{mg} / \mathrm{dL}$; o nível de colesterol sérico total abaixo de $160 \mathrm{mg} / \mathrm{dL}$; a mudança do estado funcional de indepen- dente para dependente; a ingestão alimentar inadequada; a circunferência muscular do braço inferior ao percentil 10; a prega cutânea tricipital menor que o percentil 10 ou maior que $95 .^{3}$

Medidas antropométricas e exames laboratoriais são considerados, entre a maioria dos autores, como bons parâmetros na avaliação do estado nutricional dos idosos. ${ }^{4} \mathrm{~A}$ antropometria constitui um método simples, porém com boa predição para doenças futuras, incapacidade funcional e mortalidade. ${ }^{5}$ Os valores dos exames de hemoglobina, hematócrito e contagem total dos linfócitos tendem a refletir o estado nutricional, podendo a última associar-se também à imunossenescência. ${ }^{4}$

A mini avaliação nutricional (MAN), escala já validada para classificar o estado nutricional de idosos, ${ }^{1}$ é considerada uma ferramenta de uso fácil, simples, rápido, e confiável, e permite que o risco de desnutrição seja identificado antes mesmo que as alterações clínicas se manifestem. ${ }^{6}$ Os parâmetros de referência adotados nessa escala são: "desnutrição" quando menor que 17 pontos; "risco de desnutrição" quando entre 17 e 23,5 pontos; e "bem nutrido" quando acima de 23,5 pontos. ${ }^{1}$ A MAN utiliza a soma de dois escores: um referente às alterações na 
ingestão alimentar, no peso, na mobilidade, no estado psicológico, no estado clínico e no índice de massa corporal (IMC); e o outro referente à avaliação global. ${ }^{1}$

A fratura do fêmur proximal é uma causa comum e importante de mortalidade e perda funcional no idoso. ${ }^{2} \mathrm{~A}$ incidência deste tipo de fratura aumenta com a idade devido aos aumentos do número de quedas e da prevalência de osteoporose. ${ }^{7}$ Pode ser intracapsular ou extracapsular. No primeiro tipo, estão incluídas as fraturas do colo e da cabeça femoral. No segundo, as fraturas transtrocanterianas, sendo que ambas decorrem de traumas de baixa energia. ${ }^{8} \mathrm{~A}$ desnutrição proteico-calórica é um importante determinante do resultado clínico de pacientes idosos após fratura de quadril, mas a eficácia dos programas de apoio nutricional na rotina prática clínica é controversa. ${ }^{9}$ Apesar destes fatos, pacientes idosos com fraturas de quadril raramente recebem avaliações nutricionais e intervenção adequada. $^{10}$

\section{Métodos}

Trata-se de um estudo transversal, prospectivo analítico e de abordagem quantitativa, aprovado pelo comitê de ética e pesquisa sob o CAAE 68313817.5.0000.5020. A população foi constituída de pacientes idosos internados na clínica ortopédica com o diagnóstico de fratura de fêmur proximal submetidos a tratamento cirúrgico. O cálculo amostral foi realizado com base na estimativa da média de pacientes operados nos últimos 3 anos através da fórmula para populações finitas (prevalência: 0,5; margem de erro: $5 \%$; coeficiente de confiança 95\%), selecionados conforme os critérios de inclusão: assinatura do termo de consentimento livre e esclarecido; idosos com idade acima de 60 anos; diagnóstico de fratura de fêmur proximal com indicação cirúrgica com até 5 dias do trauma e até $48 \mathrm{~h}$ da internação hospitalar. Foram excluídos pacientes que apresentaram distúrbios neurológicos e aqueles de origem indígena. Os pacientes foram submetidos a avaliação do estado nutricional através da escala MAN e coleta dos exames bioquímicos. Após a cirurgia, as complicações ocorridas foram registradas num formulário de coleta.

\section{Resultados}

Após a aplicação dos critérios de seleção, foram avaliados 20 pacientes durante o período de julho de 2017 a julho de 2018 . Foram realizadas a aplicação do questionário para coleta de dados e avaliação dos exames laboratoriais dos mesmos. Desta maneira, os pacientes foram catalogados e caracterizados quanto a idade, sexo, estado nutricional e complicações cirúrgicas. Destes, um foi excluído do estudo por perda de seguimento.

A amostra, então, foi composta por 19 idosos, de ambos os sexos, com média de idade de 70,8 anos (mínimo de 62 e máximo de 84 anos) e desvio padrão de 7,12 , sendo $68,4 \%$ do sexo feminino $(n=13)$ e $31,6 \%$ do sexo masculino $(n=6)$.
Tabela 1 Classificação do estado nutricional - índice de massa corporal

\begin{tabular}{|l|l|l|}
\hline & $\mathbf{n}$ & $\%$ \\
\hline Abaixo do peso & 3 & 15,8 \\
\hline Eutrófico & 11 & 57,9 \\
\hline Sobrepeso/obesidade & 5 & 26,3 \\
\hline Total & 19 & 100 \\
\hline
\end{tabular}

Em idosos (Lipschitz) segundo índice de massa corporal (IMC), $\mathrm{n}=19$;

Tabela 2 Classificação do estado nutricional - mini avaliação nutricional

\begin{tabular}{|l|l|l|}
\hline & $\mathbf{n}$ & $\%$ \\
\hline Normal & 5 & 26,3 \\
\hline Risco & 8 & 42,1 \\
\hline Desnutrido & 6 & 31,6 \\
\hline Total & 19 & 100 \\
\hline
\end{tabular}

Em idosos segundo a MAN, $n=19$;.

Tabela 3 Classificação do estado nutricional segundo parâmetros bioquímicos: contagem total de linfócitos e níveis de albumina sérica

\begin{tabular}{|l|l|l|l|l|}
\hline \multirow{2}{*}{} & \multicolumn{2}{l|}{ CLT } & \multicolumn{2}{l|}{ Albumina } \\
\cline { 2 - 5 } & $\mathbf{n}$ & $\%$ & $\mathbf{n}$ & $\%$ \\
\hline Desnutrição grave & - & - & - & - \\
\hline Desnutrição moderada & 10 & 52,6 & 7 & 36,8 \\
\hline Desnutrição leve & 9 & 47,4 & 10 & 52,6 \\
\hline Normal & & & 2 & 10,5 \\
\hline Total & 19 & 100 & 19 & 100 \\
\hline
\end{tabular}

Abreviatura: CLT, contagem total de linfócitos.

CTL $\left(\mathrm{mm}^{3}\right)$. Depleção grave: < 800; depleção moderada: 800-1.199; depleção leve: 1.200 a 2.000; normais: $>2.000$ e Albumina $(\mathrm{g} / \mathrm{dl})$ Normal: > 3,5; depleção leve: 3,0-3,5; depleção moderada: 2,4-2,9; depleção grave: $<2,4$.

Por meio da variável antropométrica IMC, identificou-se presença de desnutrição em 15,8\% $(n=3)$ dos idosos (-Tabela 1).

Após a aplicação do questionário MAN, a prevalência de desnutrição foi de $31,6 \%(n=6)$ e $42,1 \%(n=8)$ de risco de desnutrição (-Tabela 2).

Na avaliação do cálculo da contagem total de linfócitos, $100 \%$ da amostra demostrou variações fora da normalidade. Utilizando o parâmetro da albumina sérica, a desnutrição foi identificada em 89,4\% dos pacientes (- Tabela 3).

Observamos um alto risco relativo de complicações relacionado aos parâmetro albumina (9,39); MAN $(3,56)$; e IMC $(3,48)$ em comparação com as demais variáveis analisadas (-Figura 1).

A média de internação da população do estudo foi de 9,1 dias, tendo como o mínimo de 5 dias e máximo de 23 dias de 


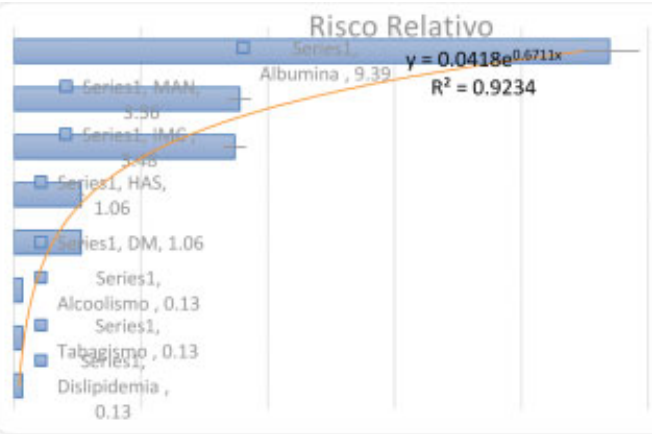

Fig. 1 Avaliação do rico relativo das principais comorbidades relacionadas com as complicações cirúrgicas na população analisada. Mini avalição nutricional (MAN); índice de massa corporal (IMC); hipertensão arterial sistêmica (HAS); diabetes mellitus (DM).

Tabela 4 Comparação entre o estado nutricional segundo a classificação da mini avaliação nutricional e a média do tempo de internação em dias

\begin{tabular}{|l|l|l|l|}
\hline $\begin{array}{l}\text { Classificação } \\
\text { da MAN }\end{array}$ & $\mathbf{n}$ & $\%$ & $\begin{array}{l}\text { Média do tempo } \\
\text { de internação/dias }\end{array}$ \\
\hline Normal & 5 & 26,3 & 5,8 \\
\hline Risco & 8 & 42,1 & 9 \\
\hline Desnutrido & 6 & 31,6 & 13 \\
\hline Total & 19 & 100 & 9,1 \\
\hline
\end{tabular}

Abreviatura: MAN, mini avaliação nutricional.

internação hospitalar, sendo que a maior média de tempo de internação foi de 13 dias nos pacientes desnutridos, quando correlacionados com a escala da MAN ( - Tabela 4).

Das complicações detectadas no pós-operatório, infecções de sítio cirúrgico estiveram presentes em $10,5 \%(\mathrm{n}=2)$ dos pacientes e ocorreram nos pacientes com risco nutricional segundo a MAN, com CTL e albumina sérica classificando-os com desnutrição moderada. Hematomas foram detectados em $26,2 \%(n=5)$ dos pacientes; destes, $5,2 \%(n=1)$ em pacientes classificados como "normal", $10,5 \%(\mathrm{n}=2)$ naque- les classificados como "risco nutricional" e 10,5\% $(\mathrm{n}=2)$ em como "desnutridos," segundo o score de MAN. Úlceras sacrais foram observadas em $5,2 \%(n=1)$ de pacientes enquadrados como "normal" e 5,2\% em pacientes classificados como "risco nutricional" pelo score de MAN. Não ocorreram deiscências de sutura ou necroses no estudo ( - Tabela 5 ).

\section{Discussão}

Os dados atuais apontam para uma prevalência de desnutrição em idosos residentes em domicílios, em nível de 1 a 15\%; em idosos internados em hospitais, essa proporção oscila entre 35 e $65 \%$. Em comparação com outros países, o risco de óbito secundário à desnutrição na 3a idade no Brasil é 71\% maior do que nos EUA e 32,13\% maior do que na Costa Rica. ${ }^{8}$

Neste estudo, foi verificado que, ao somar as taxas de desnutrição ao risco de desnutrição, a taxa de prevalência foi de $73,7 \%$ (Valor de $p$ de 0.00036 e risco relativo aumentado de 3.56, com valores mínimos de 1.55 e máximos de 8.06); $100 \%$ da amostra demonstrou variâncias fora dos parâmetros normais da contagem total de linfócitos; e em $89,4 \%$ a albumina sérica apresentou valores anormais. ${ }^{6}$

Em um ensaio clínico randomizado controlado com o objetivo de determinar se a suplementação nutricional reduz as taxas de complicações relacionadas à fratura em uma seleção de pacientes saudáveis com fraturas de quadril, foi verificado que o risco de complicações foi maior no grupo controle $(70 \%)$ do que no grupo experimental (15\%). Os autores concluíram que os suplementos nutricionais equilibrados resultam em menores taxas de complicações e mortalidade em 120 dias de pós-operatório. ${ }^{9}$

Um outro estudo evidenciou maior predisposição à mortalidade em pacientes com hipoalbuminemia $<3,5 \mathrm{~g} / \mathrm{dl}$ durante a internação, e maiores taxas de mortalidade um ano após a fratura em pacientes com linfopenia $<1,500 \mathrm{u} / \mathrm{ml}^{7}$ Segundo outros autores, a albumina sérica no pré-operatório é um forte preditor de complicações nos primeiros 30 dias de pós-operatório e está associada a maiores morbidade e mortalidade, longos períodos de internação e readmissões. ${ }^{8}$

Neste estudo foi observado que as alterações da albumina, do risco nutricional e do IMC foram estatisticamente representativos pelo alto valor de $p$ e do aumento do risco relativo.

Tabela 5 Correlação entre o estado nutricional segundo a classificação da mini avaliação nutricional e as complicações detectadas no pós-operatório.

\begin{tabular}{|l|l|l|l|l|}
\hline $\begin{array}{l}\text { Classificação } \\
\text { da MAN }\end{array}$ & $\mathbf{n}$ & Complicações & \% por classificação & \% do total \\
\hline Normal & 5 & Hematomas $(\mathrm{n}=1)$ & 20 & 5,2 \\
\hline $\begin{array}{l}\text { Risco } \\
\text { Nutricional }\end{array}$ & 8 & $\begin{array}{l}\text { Infecções de sítio cirúrgico }(\mathrm{n}=2) \\
\text { Hematomas }(\mathrm{n}=2) \\
\text { Úlceras sacrais }(\mathrm{n}=1)\end{array}$ & 62,5 & 26,2 \\
\hline Desnutrido & 6 & $\begin{array}{l}\text { Hematomas }(\mathrm{n}=2) \\
\text { Úlceras sacrais }(\mathrm{n}=1)\end{array}$ & 50 & 15,8 \\
\hline Total & 19 & & & 47,2 \\
\hline
\end{tabular}

Abreviatura: MAN, mini avaliação nutricional. 
Para Nourissat et al., ${ }^{11}$ pacientes desnutridos apresentam maior probabilidade de evoluir com complicações do que os bem nutridos, tais como: dificuldade de cicatrização da ferida, formação de fístula, infecção, aumento do tempo de internação hospitalar, diminuição do tempo de sobrevida e da qualidade de vida.

Cruz e Marimoto ${ }^{12}$ afirmam que o acompanhamento nutricional adequado garante o sucesso da cirurgia, reduzindo os índices de complicações e da morbimortalidade, assim como o tempo de internação hospitalar e infecções do sitio-cirúrgico.

Observou-se que os pacientes desnutridos apresentaram maior média de tempo de internação (13 dias) e de complicações no pós-operatório.

\section{Conclusão}

Neste estudo, os idosos em risco ou em desnutrição com diagnóstico de fratura da região proximal do fêmur apresentaram maior taxa de infecção do sítio cirúrgico e do tempo de internação hospitalar quando comparados com os eutróficos. As mensurações utilizadas neste estudo (escore MAN, albumina e contagem total de linfócitos) são bons parâmetros para avaliar o status nutricional dos mesmos.

Conflitos de Interesse

Os autores declaram não haver conflitos de interesse.

\section{Referências}

1 Guigoz Y, Vellas B, Garry PJ. Assessing the nutritional status of the elderly: The Mini Nutritional Assessment as part of the geriatric evaluation. Nutr Rev 1996;54(1 Pt 2):S59-S65

2 Alarcón T, González-Montalvo JI, Bárcena A, Saez P. Further experience of nonagenarians with hip fractures. Injury 2001;32 (07):555-558

3 Riobó Serván P, Sánchez-Vilar O, González de Villar N. [Geriatric nutrition]. Nutr Hosp 1999;14(Suppl 2):32S-42S

4 Fonseca ACE. Estado nutricional - relação com a atividade física e doenças crônicas em idosos institucionalizados [dissertação]. Covilhã: Universidade da Beira Interior; 2009

5 Maciel A. Avaliação multidisciplinar do paciente geriátrico. Rio de Janeiro: Revinter; 2008

6 Faulkner KG, Wacker WK, Barden HS, et al. Femur strength index predicts hip fracture independent of bone density and hip axis length. Osteoporos Int 2006;17(04):593-599

7 Koval KJ, Maurer SG, Su ET, Aharonoff GB, Zuckerman JD. The effects of nutritional status on outcome after hip fracture. J Orthop Trauma 1999;13(03):164-169

8 Abel RM. Nutrição e coração. In: Fisher JE, ed. Nutrição em cirurgia. São Paulo: Editora Médica Científica; 2009:546

9 Avenell A, Handoll HH. A systematic review of protein and energy supplementation for hip fracture aftercare in older people. Eur J Clin Nutr 2003;57(08):895-903

10 Sousa VMC, Guariento ME. Avaliação do idoso desnutrido. Rev Bras Clin Med 2009;7(01):46-49

11 Nourissat A, Mille D, Delaroche G, et al. Estimation of the risk for nutritional state degradation in patients with cancer: development of a screening tool based on results from a cross-sectional survey. Ann Oncol 2007;18(11):1882-1886

12 Cruz MRR, Marimoto IMI. Intervenção nutricional no paciente cirúrgico: resultado de um protocolo diferenciado. Rev Nutr 2004; 17(02):263-272 